\title{
Ultrafast dynamics of highly constrained azobenzene macrocycles
}

\author{
Chavdar Slavov ${ }^{1, *}$, Chong Yang ${ }^{2}$, Luca Schweighauser $^{3}$, Andreas H. Heindl ${ }^{3}$, Tim \\ Stauch $^{2,4}$, Herman A. Wegner ${ }^{3}$, Andreas Dreuw ${ }^{2}$, and Josef Wachtveitl ${ }^{1}$ \\ ${ }^{1}$ Institute of Physical and Theoretical Chemistry, Goethe University, 60439 Frankfurt, Germany \\ ${ }^{2}$ Interdisciplinary Center for Scientific Computing (IWR), University of Heidelberg, 69120 \\ Heidelberg, Germany \\ ${ }^{3}$ Institute of Organic Chemistry, Justus-Liebig University, 35392 Giessen, Germany \\ ${ }^{4}$ Department of Chemistry, University of California, Berkeley, USA
}

\begin{abstract}
The ultrafast photoisomerization of model azobenzene macrocycles was studied by transient absorption spectroscopy. Our results reveal a strong dependence of the dynamics and the overall molecular properties on the geometric constraints and the intramolecular strain.
\end{abstract}

\section{Introduction}

Azobenzene (AB) photoswitches are extensively used in the development of photoresponsive nanostructures due to the large geometrical change associated with the $E \leftrightarrow Z$ isomerization of the central $\mathrm{N}=\mathrm{N}$ bond [1]. While the ultrafast dynamics of single ABs is extensively studied [2], the properties of connected ABs are not well understood yet. In bis(AB)s the substitution pattern and the ensuing interactions between the individual photoswitch units modify the ultrafast photochemistry of the compounds and give rise to new properties [3]. Here, we investigate the geometrical constraints and the molecular strain that affect the isomerization dynamics of $\mathrm{AB}$ in multiunit macrocyclic compounds (Fig. 1). In the light of the wide spread construction and utilization of photoresponsive compounds, our study brings new and important insights.
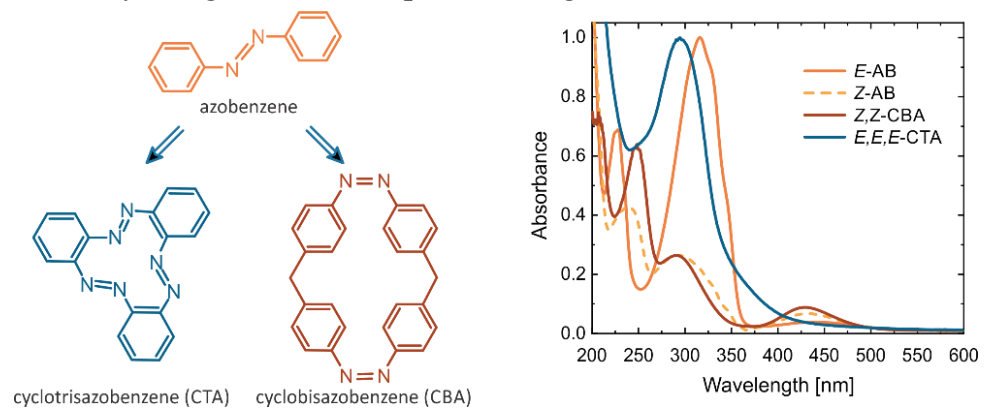

Fig. 1. left: structures of the investigated compounds; right: corresponding absorption spectra.

\footnotetext{
* Corresponding author: chslavov@theochem.uni-frankfurt.de
} 


\section{Experimental}

The transient absorption (TA) measurements ( 100 fs IRF) were performed as described previously [4]. The compounds were excited either in the $\pi \pi^{*}$ band $(\sim 300 \mathrm{~nm})$ of the $(E)$ - and $(Z)$-isomers or in the $n \pi^{*}$ band $(\sim 430 \mathrm{~nm})$ of the $(Z)$-isomers (Fig. 1). The transient absorption data were transformed into the lifetime space and presented as lifetime density maps (LDM) [5]. The study is complemented by extensive quantum chemical calculations (TD-DFT, BHLYP functional, 6-31G* basis set and a conductor-like polarizable continuum model).

\section{Results and discussion}

The dynamics of (E,E,E)-cyclotrisazobenzene (CTA) (Fig. 2) differs significantly from that of a pure monomeric $\mathrm{AB}[6]$. The ultrafast relaxation $(150 \mathrm{fs})$ of the initially excited $\pi \pi^{*}$ state does not lead to the build-up of the typical $n \pi^{*}$ excited state absorption (ESA), but is associated with partial recovery of the ground state bleach (GSB). With the cooling of the vibrationally hot molecules (7 ps), the GSB is completely recovered. This indicates that CTA dissipates the excitation energy as heat and does not undergo $E \rightarrow Z$ isomerization.
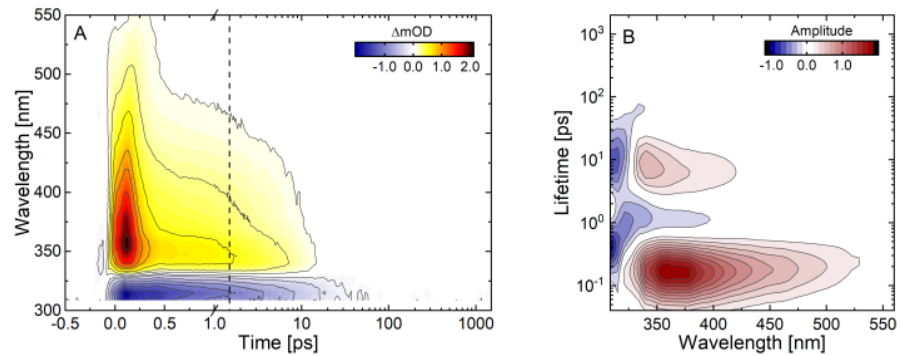

Fig. 2. Transient absorption data (left) and LDM (right) illustrating the ultrafast dynamics of $(E, E, E)$-CTA after $\pi \pi^{*}$ band excitation.

Our theoretical calculations showed that the strong constraint on the CNNC dihedral angle and the high density of states in CTA provide a highly efficient and barrierless relaxation pathway from the excited $\pi \pi^{*}$ state to the lowest $n \pi^{*}$ state. On the $n \pi^{*}$ potential energy surface (PES), we find that the typical $\mathrm{AB}$ conical intersection (CI) is shifted due to the molecular strain in CTA from $90^{\circ}$ to $110^{\circ}$ and sloped towards the initial $(E, E, E)$-isomer. This explains the ultrafast, completely non-photochemical relaxation of excited CTA. Therefore, similarly designed macrocycles can be employed as efficient UV absorbers.

We further investigated the macrocyclic compound cyclobisazbenzene (CBA) (Fig. 1), which is locked in the $(Z, Z)$-isomer due to geometric constraints [7]. The ultrafast dynamics of $(Z, Z)$-CBA (Fig. 3) is similar to that of monomeric (Z)-AB. It proceeds from the initially excited $\pi \pi^{*}$ state to the lowest $n \pi^{*}$ state, where a CI to the ground state (GS) is encountered, which leads to a partial recovery of the initial $(Z, Z)$-isomer and formation of the $(Z, E)$-isomer photoproduct. Despite the highly constrained structure of CBA and in contrast to CTA, the presence of a nanosecond non-decaying component in the transient absorption data indicates a relatively high $Z \rightarrow E$ isomerization efficiency. Nevertheless, the $(E)$-isomer of CBA is not stable and relaxes back on the millisecond timescale.

GS geometry optimization shows that the $(Z, Z)$-isomer is the most stable form of CBA. This is due to the only minor distortion of the CNNC dihedral angle as compared to monomeric $(Z)-\mathrm{AB}$. Contrariwise, the CNNC dihedral angles of the $(Z, E)$ - and the $(E, E)$ isomers of CBA are strongly distorted which leads to higher GS energies. Strain analysis [8] of CBA confirms that despite the geometric constraints, the strain in the $(Z, Z)$-isomer is 
negligible, while in the geometrically distorted $(Z, E)$ - and the $(E, E)$-isomers it is very high. The latter is also the reason for the high $E \rightarrow Z$ relaxation rate.
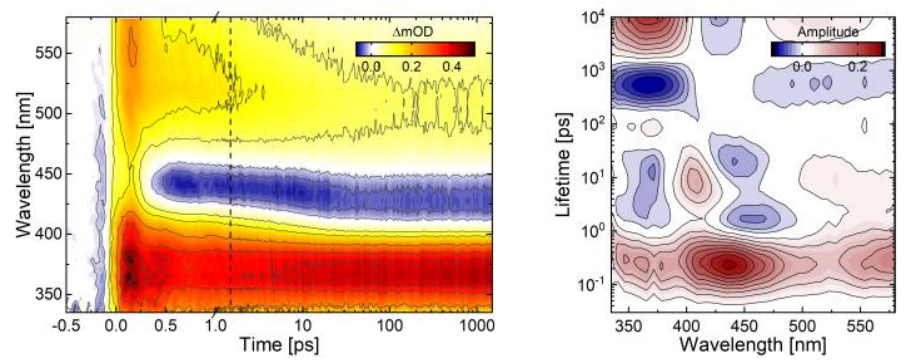

Fig. 3. Transient absorption data (left) and LDM (right) illustrating the ultrafast dynamics of $(Z, Z)$-CBA after $\pi \pi^{*}$ band excitation.

Geometry optimization was also performed for the first three excited states (ES) of CBA. The $\mathrm{S}_{3} \mathrm{PES}$ is relatively flat in the $0-50^{\circ}$ range of the CNNC angle, after which an energetic barrier arises. On the $\mathrm{S}_{3} \mathrm{PES}$, at $\sim 50^{\circ}$ of the $\mathrm{CNNC}$ angle a crossing is located with the PES of the energetically close $\mathrm{S}_{2}$ state. The $\mathrm{S}_{2}$ PES also shows a rising energetic barrier for $\mathrm{CNNC}$ angles larger than $50^{\circ}$, but provides a quick relaxation pathway to the lowest ES $\left(\mathrm{S}_{1}\right)$ in the direction of $0^{\circ}$ of the CNNC angle. The PES for the optimized $\mathrm{S}_{1}$ structure resembles that of monomeric AB. The typical CI to the GS is located at $\sim 80^{\circ}$ of the $\mathrm{CNNC}$ dihedral angle, which explains the relatively high $\mathrm{Z} \rightarrow \mathrm{E}$ isomerization in CBA. It appears also that the higher ES relaxation rate of CBA is due to a steeper slope towards the GS CI on the $\mathrm{S}_{1}$ PES. Finally, the possibility of concerted $Z, Z \rightarrow E, E$ isomerization in CBA was explored. Although such a scenario cannot be completely ruled out, we find it unlikely due to the presence of high energetic barriers towards $90^{\circ}$ of the $\mathrm{CNNC}$ dihedral angle on all ES PES including $\mathrm{S}_{1}$.

In conclusion, our investigation of the CBA and CTA reveals that the ultrafast dynamics and the ensuing photoisomerization quantum yields in photoresponsive macrocyclic systems is very sensitive to the geometrical constraints in the molecule and the intramolecular strain along the relevant molecular coordinates. Therefore, these effects should be carefully considered in the design and development of complex macrocycles as they have a critical impact on the PES structure and the overall molecular properties.

\section{References}

1. H. Dürr, and H. Bouas-Laurent, Photochromism: Molecules and Systems (Elsevier, 2003).

2. H. M. D. Bandara, and S. C. Burdette, Chem. Soc. Rev. 41, 1809 (2012).

3. C. Slavov, et al., PCCP, 17, 14795 (2016)

4. C. Slavov, et al., PCCP, 17, 14045 (2015).

5. C. Slavov, H. Hartmann, and J. Wachtveitl, Anal. Chem. 87, 2328 (2015).

6. C. Slavov, et al., ChemPhysChem, 18, 2137 (2017).

7. A. H. Heindl, L. Schweighauser, C. Logemann, H. A. Wegner, Synthesis, 49, A$\mathrm{H}$ (2017).

8. T. Stauch, A. Dreuw, Acc. Chem. Res., 50, 1041 (2017). 